Author: $\quad$ Bruce E Perrott

School of Marketing, University of Technology, Sydney PO Box 123 Broadway, NSW 2007

Phone contact; 0295143524

Fax 0295143535

E-mail; Bruce.Perrott@uts.edu.au

Track: Knowledge and Learning

Word Count: 6239 


\section{KNOWLEDGE MANAGEMENT FROM AN INDUSTRY PERSPECTIVE}

\section{Summary}

Strong forces of competition and globalisation have created awareness and an urgency to focus how an organisation controls and nurtures its intellectual capital. The knowledge concept and its management have gained currency and momentum as technology has enabled thoughts and ideas to be more easily produced and distributed. It gained considerable discussion in the late 1990's in the management literature. With the increased application of recent technologies such as the Internet, customer relationship management and advanced software capabilities, it has been suggested that the time has come for a debate on a new paradigm for knowledge management. As a contribution to this debate, this paper will examine exploratory research conducted in the Australian private hospital industry with a view to better understand issues related to knowledge management from an industry perspective. 


\section{A Perspective on Knowledge Management}

Knowledge management (KM) is not a new concept. Beyond its role in ancient world history, it came into main stream relevance with the invention of Johann Gutenberg's printing technology in about 1455 .With the increase in printed books and manuscripts came the development of libraries which became the critical source of knowledge for many people for the first time. In the seventeenth century there was a rapid expansion of learning and knowledge through newly formed societies which had the charter of disseminating new thinking and knowledge through journals (Wiegand 1994).

The next major change impacting on dimensions of information and knowledge capture, storage and distribution was the introduction of computers. Digital words were captured and shared between computers across wide geographical distances. This trend gathered momentum with the spread of the Internet. The opportunities for innovation and diffusion in knowledge use gathers momentum as information technology advances.

In the 1990's senior managers began to talk about knowledge management as they began to realise that the foundations of modern economies had shifted from natural resources to intellectual assets. Networked computers provided the capability to address how knowledge may be codified, stored and shared, practically and economically (Hansen et al 1999). One estimate from this period suggested that threequarters of the Fortune 100's total market capitalisation was represented by intangible assets such as patents, copyrights and trademarks. Hence the responsibility of managing these important company assets must be the concern of senior managers as well as the corporate legal staff (Reitzig 2004).

One indication of recent renewed interest knowledge management was in Australia. In November 2005, one hundred business and government leaders were brought together in Melbourne to focus on formulating effective approaches and best practice in valuing, reporting and managing knowledge resources within organisations. They promoted the principle of knowledge capital reporting and endorsed the creation of a professional society of knowledge economics which is dedicated to improving the quality of information related to the identification, management, measurement and reporting of knowledge economics for the benefit of industry and the community (Gap Congress 2005).

To some, knowledge management is seen to be a logical extension to three basic business trends (Guptill 2005);

a) increasing amount of digitised information data that is available, $24 \times 7$

b) globalisation of business such that production can occur anywhere in the world as it is the knowledge that is the true source of competitive advantage

c) growing complexity of business requires that new business processes will deliver; 'the right information at the right time' so as to ensure accountability and reduce the risk of mistakes

When exploring this area of knowledge, it is useful to make a distinction between various use graduations of terminology discussed in the literature. Data is often described as the base platform in the knowledge hierarchy and is defined as facts and 
statistics either historical or derived from experimentation or calculation (Geddes and Grosset 1999). Information is the next step in terms of value and has been considered as, 'systematically organised data' (Meadows 2001). Knowledge has been seen as 'actionable information' thus more effectively assisting in the decision making processes within the organisation. Wisdom is often seen as the highest dimension on the knowledge tree where it is possible to act appropriately in a given situation with a strong element of ethical judgement ( Jashapara 2004).

\section{What is Knowledge Management?}

In more recent times a new focus of interest has emerged post industrial times known as the "knowledge economy" ( Drucker 1992). The management of knowledge has gained interest from both academics and practitioners with the realisation that knowledge holds the key to organisational growth and development. Research and publications have emerged from different disciplines reflecting the wide impact of this interest area on numerous functions and at different levels of the business. Some have conveniently attempted to organise contributions into those that have an information based approach, while others have looked more at the human side of knowledge creation, sharing and management.

It has been suggested that knowledge management as a field of study will gain considerable momentum through dialogue and debate with multiple disciplines. It has also suggested that this field of study will yield rich rewards as it moves into a new paradigm of work (Jashapara 2004).

The literature contains many definitions of knowledge management. A few are listed here in order to observe difference in perceptions of scope and emphasis;

"Knowledge management draws from existing resources that your organisation may already have in place- good information systems management, and human resource management practices” (Davenport and Pusak 1998)

“.. any processes or practice of creating, acquiring, capturing, sharing and using knowledge, wherever it resides, to enhance learning and performance in organisations" (Swan et al 1999)

"... all methods, instruments and tools that in a holistic approach contribute to the promotion of core knowledge processes” (Mertins et al 2000)

"Knowledge management is the identification, storage, protection of knowledge for future operational and strategic benefit of the organisation- this may be implicit or explicit” (Perrott 2006)

The latter definition is used to guide the discussion of this paper as it contains a number elements considered essential in helping the reader to conceptualise the scope and dimensions of knowledge management in organisations. Firstly, it distinguishes between operational and strategic knowledge. Strategic knowledge being knowledge which is essential to major decisions an organisation must make to capitalise on priority opportunities and successfully overcome major threats. Operational 
knowledge is concerned with the day to day running of the business. Secondly, it recognises that knowledge contained in an organisation may be implicit ( that is remaining in the domain of the individual), or explicit (knowledge that is available for use throughout the organisation). One critical dimension of contemporary knowledge management is the sensitive but critical issue of when, if and how implicit knowledge should be made explicit and available for wider use throughout the organisation. The third benefit of this definition is that it recognises knowledge management as process rather than an occasional or one-off event. Ongoing and continuous process will be essential in actioning knowledge creation in vital areas of knowledge deficiency (refer to Drew’s (1999) knowledge classification framework outlined below in this paper)

\section{Dimensions of knowledge}

There is considerable debate in the literature about the various types and dimensions of knowledge. The distinction between tacit and explicit knowledge receives considerable attention. Tacit knowledge is that held in the minds of individuals while explicit knowledge is that externalised and shared with others. It has been suggested that there are four modes of interaction between these two forms of knowledge (Polanyi 1967);

- From tacit knowledge to tacit knowledge: the process of 'socialisation' through shared experience and interaction

- From explicit knowledge to explicit knowledge: the process of 'combination' through reconfiguring existing knowledge such as sorting, adding, recategorising and reconceptualising explicit knowledge can lead to new knowledge

- From tacit knowledge to explicit knowledge: process of 'externalisation' using metaphors and figurative language

- From explicit knowledge to tacit knowledge: the process of internalisation through the learning process

One author describes the knowledge management process as necessarily loose and collaborative because the human qualities of knowledge such as experience, intuition and beliefs are not only the most valuable, but also the most difficult to manage and maximise (Daveport and Prusak 1998). Hence the knowledge management process integrates theories from at least four distinct fields; theories about organisational culture, organisational structures, organisational behaviour and knowledge based systems leading to theories about knowledge support infrastructures (Baskerville and Dulipovici 2006).

More recent research emphasises the importance of context in the knowledge conversion process (Ancori, Bureth and Cohendet 2000) and that knowledge should be seen as a cultural process situated in and inextricably linked to the material and social circumstances in which it is produced and consumed (Hassard and Keleman 2002). A balanced environment of power, control and trust is seen as essential condition for a successful knowledge oriented culture, Allee (2003) suggests that if people do not trust each other, they do not exchange knowledge and ideas. Here trust helps build and sustain valuable networks and rewarding relationships while a lack of trust erodes knowledge leadership, creation and transfer. 
The knowledge management process is seen to begin with the formulation and implementation of strategies for the construction, embodiment, distribution and use of organisational knowledge. Other strategies include those for the basic management functions to monitor and measure the knowledge assets and processes (Quintas et al 1997).

Organisation context is critical to effective knowledge management. The ideal structure has been described as ' $\mathrm{N}$-form' rather than the traditional 'M-form'. M-form is a hierarchical structure where communication is primarily vertical with top management as the critical layer and the competitive scope is based on economies of scale and diversification. By contrast in the $\mathrm{N}$-form, communication is lateral where middle management is the critical layer and competitive scope focuses on specialisation (Hedlund 1999).

\section{Relevance of knowledge management}

The renewed focus on the management of knowledge can be explained by the increasing demands placed by the operating environment of contemporary organisations. Knowledge becomes the critical currency in determining outcomes in a competitive and demanding world. Pressures to bring about the effective management and organisation of knowledge have been summarised by one author as follows (Perrott 2006);

* Rapidly changing and turbulent operating environments - hence the need to generate, share and leverage new knowledge about rapidly changing opportunities and threats

* Stakeholder demands and expectations- hence the need to understand the nature and location of knowledge capital within the organisation so that it may be protected and leveraged

* Corporate governance- the need for transparency and accountability of knowledge assets by responsible caretakers

* Accountable risk management- having up to date knowledge about the nature and level of risks to the ongoing satisfactory performance of the operation

* Replication of performance- having comprehensive knowledge of an operation on critical factors that are necessary to ensure the organisation will perform time after time with a high level of probability and to the satisfaction of key stakeholders

What may have been considered once to be an optional luxury in terms of corporate management capabilities, knowledge management can be expected to take a front seat in priority essentials going forward. Awareness and conscious control of the knowledge management dimensions, protocols and procedures are most likely to become a base requirement of accountable management. In addition to gaining and managing knowledge, some authors have mentioned that managers should also be conscious of the need to shed knowledge as the need arises (de Holan, Phillips and Lawrence 2004). 


\section{Industry Knowledge}

Much of the knowledge literature tends to focus at the organisational level. However, challenges of knowledge management have been seen to be the establishing and optimising the information-knowledge balance appropriate to a company or industry (Blumentritt and Johnston 1999). In recent times there have been more occasions for organisations to collaborate for mutual benefit. Some say that self sufficiency is becoming increasingly difficult in a business environment that demands strategic focus, flexibility and innovation and that many firms enter alliances with specific learning objectives (Inkpen 1996).

Some researchers have even proposed industry level knowledge management theory that will lead to a better understanding of how the routine day to day activities of firms and support organisations that make up an industry group can be coordinated (Johnston and Gregor 2000).

The need for company liaison at an industry level can be seen by the increasing number of strategic alliances. Some previous researchers have explored the aspect of knowledge transfer within such alliances with the view to measure knowledge movement based on the changing pattern of patent portfolios of alliance members (Mowery et al 1996). However, managers are finding it increasingly difficult to capture value from such alliances. This has prompted some authors to propose a model that describes the knowledge resource exchange between alliance partners (Parise and Henderson 2001).

This paper will review the research findings of an industry study which probes senior manager's opinion as to future successful performance parameters, future industry challenges and perceived ingredients for future success. The objective will be to gain insights into knowledge areas of relevance in the future operating environment of the Australian private hospital industry whilst gaining insights to knowledge management practice in an industry setting.

The research approach taken for this project was by exploratory qualitative in-depth interviews of about one hour each with 28 senior industry executives. One or two researchers were present at each interview usually conducted at the respondent's place of business. Data was collected via note taking and audio recording. Respondents were selected on the recommendation of industry members and on the basis of their willingness to participate. Interviewees were asked to respond to a list of prepared questions about the industry. Data analysis was undertaken using the software program ENVIVO Power version, revision 4.0. A limitation of the research is the small sample of 28 senior executives, impacting on the generalisability of the results and conclusions.

\section{Site Industry Background}

The Australian health care system has been described as having the distinguishing characteristic of being a mixed economy comprising a tapestry of programs funded by federal and state government, private health insurance, government owned 
institutions, private medical practice, private for-profit and not for profit institutions, corner shop pharmacies and large publicly listed and private corporations (Foley 2000).

This review will focus on health care delivered in Australian private or non public hospitals. There were 532 private hospitals in operation during 2004-05. The number of available beds was 26,424 with total patient separations of 2.8 million. About four in ten hospital patients were admitted to private hospitals in 2004-05. Equivalent full time staff at private hospitals was 48,544. Patient separations covered by private hospital insurance amounted to $78 \%$. Total income generated at these hospitals totalled AUDS\$6,624 million. Net operating margin for acute and psychiatric hospitals was 7\%, a contrast to the 19\% realised for free standing day hospital facilities. (ABS 2006).

\section{Industry Attractiveness Overview}

In order to understand the context of the private hospitals industry, it is useful to undertake an analysis of the key forces and dynamics at a point in time. One framework that can be used to structure this process is the five forces model (Porter 1980). This has been seen as being one of the most significant contributions to the understanding of industry dynamics and market power through its structure-conductperformance framework (Rumelt et al 1991, Hoskisson 1999).

Here, the competitive landscape is at the central point of focus which examines the nature and details of how the key firms compete in the industry. Potential entrants and likelihood of entry to and exit from the industry are shown at the top of the diagram. The role and threat of substitute products and service is considered at the base of the figure. The impact and bargaining power of buyers is positioned at the right side of the chart. The impact and bargaining power of suppliers to the industry is positioned at the left side of the chart. This paper will use the five forces framework as the basis for review and discussion of the Australian private hospital industry.

This section will briefly summarise the circumstances of each of the five industry elements or forces and make a summary assessment in terms of each areas relative attractiveness; $\because$ or unattractiveness $; \circ$ to industry members.

\section{Industry Core}

Through progressive rationalisation and consolidation, the for-profit sector of the industry theoretically operates as an oligopoly with three main companies owning $92 \%$ of the market in private hospital beds. In the not-for-profit sector there are only four main groups operating in the industry. Under such a market structure one would expect to see intense rivalry manifest in terms of aggressive marketing activity in order to maintain or build market shares.

However in the case of the private hospital industry, each company and hospital has its unique positioning near key markets and referring doctor networks. This unique positioning limits the competitive rivalry between the companies in the industry. It 
also has the effect of containing the level of marketing spend and minimising the necessity to reduce health service prices in order to attract customers.

The negative effect of limited competitive rivalry also means that it would be more difficult for an individual company to build market share through aggressive marketing similar to what could happen in most other industries when markets are not as protected. This limit on the level of competitive rivalry also has longer term benefits to shareholders of the three key companies. They benefit from better profit margins and logically larger dividend distributions over time. It means also that yields on the asset of private hospital beds is somewhat protected from the ravages of competitive business and marketing strategies.

It should be mentioned that even though competition within this industry may be at moderate levels, conditional competition for certain patient revenue still occurs from operators outside the industry such as day care hospitals, public hospitals and the not for profit sector.

Another favourable dimension is the outlook for industry growth. Due to the ageing Australian population outlined in this paper and the increasing awareness of the need for high quality health care, the demand for health services is likely to continue to grow in the foreseeable future. Hence the return to shareholders of companies in the private, for profit industry should be maintained provided costs are contained and market shares are not lost to competing industries such as the not for profit or day care.

The search for growth may involve varying degrees of diversification, whether it be into closely associated health services not currently being offered by the private hospital sector or moving into the closely associated industry of aged care. Alternatively, for those more adventurous, diversifying into the emerging wellness industry.

One author has identified the birth of a new and substantial industry which runs separate but parallel to traditional health care industries such as the one under review (Pilzer 2002). He sees the beginning of a huge industry described as the 'Wellness Industry". Whilst he sees existing health care as reactive, the wellness industry will be proactive and dedicated to preventing people becoming customers of the healthcare industries. Depending upon how healthcare industry members define their business, wellness could mean a whole new wave of growth opportunities for stakeholders.

In summary, it can be stated that the competitive environment for private hospital members is generally favourable;

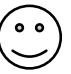

\section{Entrants}

There are moderately high barriers to entry in this industry. High capital costs and the difficulty in finding and building new hospitals in locations close to viable markets presents a formidable hurdle for potential new entrants to the industry. It has been suggested that the current capital costs of building and commissioning a hospital bed is about $\$ 450,000$ (Grier 2004). There is also the issue of obtaining the necessary bed 
licences in sufficient numbers from government authorities. In addition, the challenge of creating a network of effective referring doctors would take time and effort, as well as increasing the cost of entry.

Entry on a relatively large scale could take place through acquisition. Such an entrant could be a company with substantial funds to invest into diversified geographical markets. An example here may be a large US health care company. Another example may be a health insurance company wanting to integrate forward from insurance to health service provision. Industry entry under these circumstances cannot be classified as a negative threat to shareholders as it could be assumed that such an entrant would need to pay a premium to encourage shareholders of the target organisation to sell controlling interest in a private health care company.

Following this rationale it can be said that the threat of new entrants impacting on industry players in a negative or damaging way to shareholder assets, and returns is low; $-\circ$

\section{Substitutes}

Taking the definition of substitutes as alternative health services offered by organisations outside the core industry, the following summarises each element which was discussed above in the main text under "Substitute Products and Services";

- Not for Profit hospitals: $\quad$ Medium to High threat depending on hospital location

- Public Hospitals: $\quad$ Low to Medium threat depending upon location and market demand

- Day Care hospitals: Medium threat depending upon type of service and location

- Technology as a Substitute to private hospital care (eg e-health): Low threat

- Reduction in need/demand for private health services: Nil threat

Following this rationale it can be summarised that the combined threat of substitutes impacting on industry players in a negative or damaging way to shareholder interests or assets, and returns is low. Hence this element is favourable to industry members

\section{Suppliers}

This variable has the potential to be most critical for members of the for profit private hospital industry in managing viable hospital units. Individual organisations rely on key groups to supply quality and timely services and products to the various hospital locations. Each of the main supplier components are summarised as follows;

Medical practitioners:

Having a strong network of referring doctors is a fundamental prerequisite to viability and success. They also need to be positively supportive of each hospital and company. Case histories show that non support of 
doctor groups can lead to serious underperformance of both individual hospitals and company groups in this industry ( Grier 2004).

Nursing staff: $\quad$ Also a critical component of the private hospital operation. In Australia they are well organised in terms of union representation and enjoy a strong positive image with the press and the public. Nurses therefore have strong collective bargaining power with private hospital groups. Hence they are in a strong position to negotiate conditions and pay structures.

Consumable Medical supplies: $\quad$ Most consumables used in private hospitals are supplied by industries that are oligopolies in structure. Hence individual supplying companies can usually exercise some control over price and supply conditions. Prostheses are a costly component of some medical treatments and are supplied by a limited number of suitable manufacturers. It has been said that supplying manufacturers are specified by medical practitioners in $20-40 \%$ of cases that require the use of a prothesis (Grier 2004).

Medical Equipment: $\quad$ Over time the private health industry has become increasingly dependent on advancing technology by way of high capital cost equipment which is used for diagnostics and treatment of medical conditions. There are a limited number of companies supplying such equipment. Hence there is limited opportunity for competitive buying by hospitals. Rapidly advancing technologies which are an integral aspect of equipment also infers that purchase prices for such equipment will be relatively high. This is due to the need to recuperate research and development costs and the lack of direct competition in each equipment category.

In summarising the impact of the suppliers, it can be said that they have the potential to impact on industry players in a negative or damaging way. Even if this influence is not activated, they hold a latent power which can be used in negotiating conditions of supply to industry members 0

\section{Buyers}

The role of buyer in this industry structure is complicated by the fact that private hospital patients rely heavily on two key groups which have a powerful role to play in the specification, supply and payment of the health services provided.

The first group is the referring doctors that specify and recommend not only the procedure to be followed but also the location or hospital where the procedure will be administered. As most private patient separations (81\% in 2002-03 (ABS 2004).) carry private health insurance, the health insurance funds play a key role in deciding how much will be paid to a hospital for a particular service and how much gap the patient will need to pay for the service. The roles that each of these two key groups play, has been discussed under the appropriate categories above. Australian private hospitals rely heavily on the health insurance funds for the payment of its services. In 2004, they paid for 77\% of available bed days (Deutsche Bank 2005).

In summarising, the role of patients in isolation to other influencing factors, it can be said that their ability to impact on industry players in a negative or damaging way to 
shareholder interests or assets and returns is low. Hence this element is favourable to industry members; $\odot$

\section{Industry Overview}

Figure A summarises the status of the private hospital industry in terms of unfavourable and favourable influences impacting upon its operations.

Analysis of this industry shows that, overall it is a favourable operating environment for industry members, with four assessments being summarised as favourable and only one as being potentially unfavourable. The unfavourable rating was allocated to the supplier sector because of the potential impact of the use of power by the various suppliers to industry members. Here suppliers could dictate both terms and cost of supply thus impacting upon industry member viability. This negative impact effect has been sighted as an important reason for the poor performance of the previous Mayne group (MRE, a) 2003). Indeed, long term favourable supply relationships for industry members has been seen to be a prerequisite for long viability of private hospital industry members (MRE, a) 2003).

\section{Figure A Industry Attractiveness Summary}

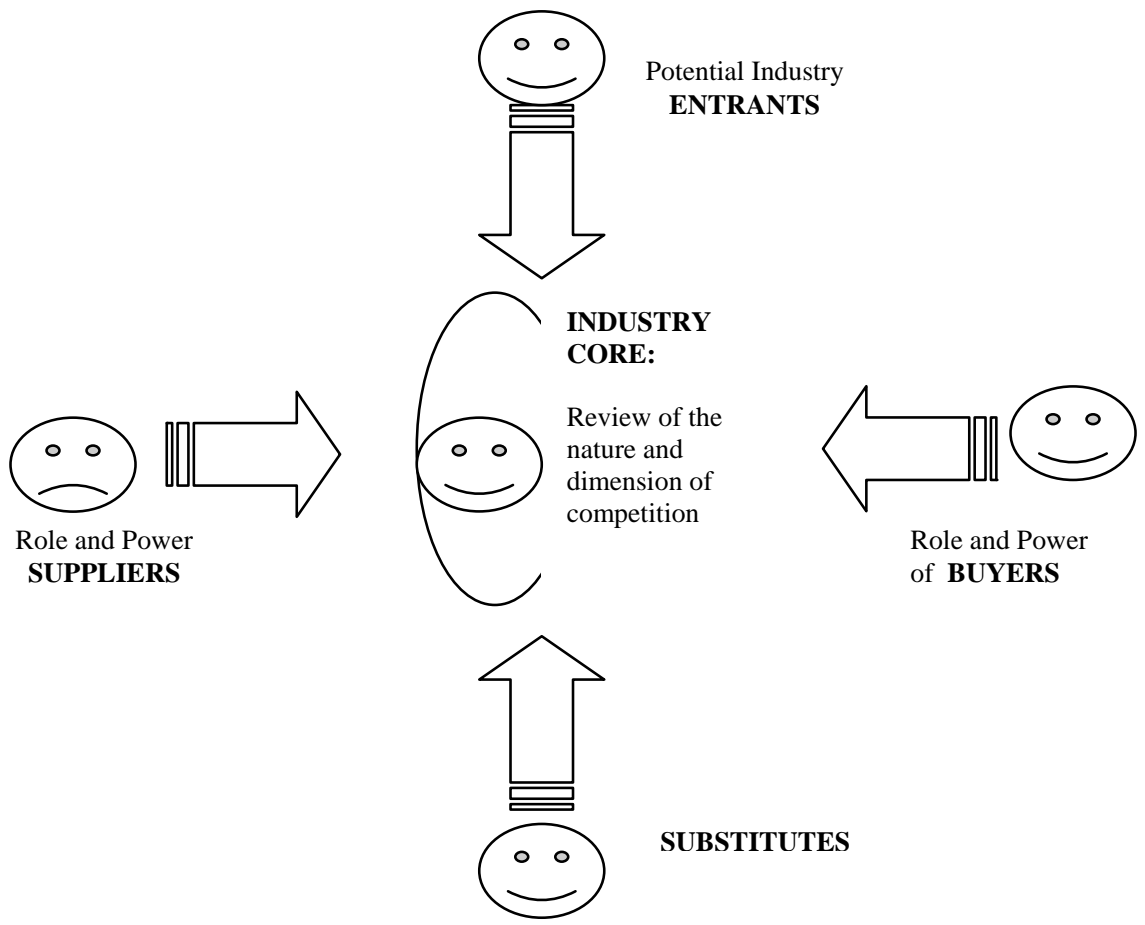

Adapted from Michael Porter (1980) 


\section{Industry Interviews}

The first question asked in the in-depth interviews provides a context and understanding of the current position of the Industry and hence insights to sectors of knowledge interest to industry stakeholders; 'What have been the most significant changes in this industry over the past decade?'. Weighted responses are listed below with percentage of respondents mentioning this factor as being significant shown in brackets

1 Federal Government introduction of private health insurance incentives to boost fund membership (79\%)

$2 \quad$ Strong growth in day surgery procedures (54\%)

3 For-profits industry consolidation (54\%)

$4 \quad$ Federal Government support for private hospitals to support the stressed public hospital system (46\%)

5 Role of the health insurance funds and aggressive negotiation with private hospitals (43\%)

$6 \quad$ For-profit hospitals now recognised as an industry with growth and investment opportunities (39\%)

$7 \quad$ For-profits: change from a 'cottage industry' to a well regarded health service provider with professional management $\quad$ (36\%)

8 Case mix in private hospitals with a reduction in bed stay time

$9 \quad$ Consumer expectations regarding type/range and quality of services provided (25\%)

10 Not for profits; Centralisation/corporatisation and focus on financials (21\%)

As explanation to this set of responses, the Federal Government were aware of the problems that were being caused by a public hospital system that was not able to cater for the high levels of demand for health services in the electorate. In an effort to partially satisfy this excess demand, they elected to build the incentive for the Australian people to become members of private health insurance funds. It should be noted that private health insurance membership had fallen to an all time low ( $30 \%$ of families were members in 1998). This is significant as $78 \%$ of patient separations in private hospitals are covered by the health insurance funds. Hence a rebate scheme was introduced to encourage more people to take out private health insurance. By 2005, private health insurance family membership had increased to $43 \%$ (Perrott 2005). 
This renewed support for private hospitals had a positive effect in terms of public acceptance of the quality of health services offered by private hospitals. It also encouraged investment and development in this industry. The increased attractiveness was also responsible for an ongoing series of mergers and acquisitions to the point that the for-profit sector of the industry has been rationalised down to only one main company (Ramsay Health Care) which held about $30 \%$ of the total private hospital market in 2005 (Low and Prior 2005).

Rationale for the second question relates to members insights of forward critical knowledge areas necessary for successful industry participation and successful survival; ' Looking forward what do you think will be the most significant challenges and issues industry members will need to meet during this time?'. Weighted responses are listed below with percentage of respondents mentioning this factor as being significant shown in brackets.

1 Issues related to hospital staff'; supply/costs/mix/training (82\%)

2 Technology/prostheses/drugs; returns/costs/returns (71\%)

3 Handling Federal and State government; conflicting policies/control/change (61\%)

4 Managing the increase in demand for health services and changing patient profile (57\%)

5 Health insurance membership; profile/costs/model/numbers

6 The increasing cost of health services and the implications of this (43\%)

$7 \quad$ Changing strategies for health service delivery; a community based model $(42 \%)$

8 The impact of continued industry consolidation and change (39\%)

$9 \quad$ Negotiating with the health insurance funds and managing the gap between payout rebates and the costs of service

10 Public-private hospitals; roles/balance/cooperation (36\%)

11 The future supply of hospitals and the lack of Greenfield developments (29\%)

12 Needed attention to IT and ehealth development and enhancement (14\%)

A key issue frequently brought forward by respondents was the fact that industry operating costs have been increasing at approximately double the rate of inflation. Total private hospital expenditure increased by $7 \%$ in FY 2005, down from $8 \%$ in 2004. Expenditure increases are seen to be a result of increasing complexity of hospital procedures and the increasing cost of inputs such as supplies, drugs and 
prothesis. Although wage costs are often sighted as a major cause of expenditure increases, analysis shows that wages as a percentage of total private hospital expenditure has progressively decreased from 59\% in FY 1998 to 50\% in 2005.

Industry income has increased at approximately the same rate as expenditure since 1999, leaving margins stable at about 5\% (Low and Prior 2005). The relatively low margins and lack of margin growth may partially explain why there have been very few new private hospitals built in recent years (described as Greenfield development by Industry executives). The CEO of one major group suggested that the high capital costs of building and fitting out new hospital developments was another reason for the low number of new hospital buildings. Instead, there has been more focus on hospital extensions and refurbishment work (described as Brownfield development by Industry executives).

Private hospitals are controlled by both State and Federal governments. Both have radically different philosophical positions on how health services should be managed. Hence industry members find it difficult to respond effectively to policy changes initiated by the two levels of government. This also impacts on the role and balance of private and public hospitals in the delivery of health services to Australians. State Labor governments generally support the allocation of resources to the public hospital system. By contrast, the Federal Coalition government favour strategies to support a strong private hospital system operating in tandem with the public hospital system.

Rationale for the third question relates to members insights to forward critical knowledge areas which will drive industry members capabilities. This involves having knowledge of what are the perceived ingredients that will enable industry members to be successful in the operating environment expected in the future; 'List what you consider as the key ingredients for industry members to be successful in the years ahead? Weighted responses are listed below with percentage of respondents mentioning this factor as being significant shown in brackets.

1 Strong hospital operational management; quality/costs/staff/profit (68\%)

2 Progressive business strategy development including innovation

3 Workforce development and management (61\%)

$4 \quad$ Cost management, discipline and control $\quad((36 \%)$

6 Strategic marketing; branding/new markets/new products (36\%)

$7 \quad$ Close liaison with medical staff including business strategy (36\%)

8 Liaison and communication across industries; For-profit \& not for profits/health funds/public hospitals (32\%)

9 Effective negotiation with the health insurance funds 
Many respondents believed that future success in the industry will rely upon having the knowledge of the dynamics and complexities of the hospital environment. From this will come the capability to manage private hospitals so that agreed objectives are achieved and key performance parameters are adhered to, thus meeting the expectations of key stakeholders. Responses indicated that knowledge of how best to manage hospitals in these circumstances will need to embrace both the strategic and operational dimensions of the private hospital business. Strategies will need to be coordinated across the numerous disciplines and functions to achieve agreed milestones whilst keeping within cost and expenditure targets.

Knowledge on how to manage hospital complexities at the operational level is seen to be critical in an operating environment which is often described as complex and turbulent. Given these demanding operating conditions, the capability of subordinate managers will become more important.. Hence the perceived need for effective and ongoing management development and training programs which focus on key areas of knowledge and skills. This is seen to be a challenge because of the diverse range of disciplines that need to work together to achieve a commercial result.

Applied knowledge of strategic marketing was seen to be important in future years. Marketing knowledge will need to be applied to issues such as brand management, market segmentation, and the development and positioning of new health services to meet the changing needs of private hospital patients. Improved effectiveness in this area would assist in the move toward the perceived need for a more community and patient centric orientation of private hospitals.

Other important knowledge areas were mentioned around the application of new technologies, IT and ehealth. Knowing when to introduce these key forces is seen to have important implications for private hospital efficiency and effectiveness.

\section{Knowledge implications for managers}

Following on from this research and analysis, what are the knowledge implications for managers in the private hospital industry? What are the implications in terms of future areas of knowledge management practice and its application to successfully survive in this industry?

This research shows that there will be a need for a concentrated focus on profit margin management. There will be strong pressures from the health insurance funds to contain prices charged for hospital services. It is important to note that the health 
insurance funds covered 78\% of patient separations in 2004-05 (ABS 2006). This places constraints on the prices charged for private hospital health services and therefore impacts on revenue receipts. There are also very strong pressures on the private hospital cost dimension by the key supplier groups of products and services to the industry, namely medical practitioners, nursing staff and medical equipment suppliers. Hence a critical and ongoing applied knowledge focus will be on how to run hospital operations to acceptable quality standards with less costly resources and with constant operational innovation. Another dimension of margin management will be to know how to actively manage case mix by increasing the proportion of services with higher profit margins.

Strategic management knowledge and skills will become more critical as pressure builds to find future revenue and growth opportunities. These opportunities will come through varying combinations of; the successful introduction of new products and services, increased penetration to high priority market segments, or accessing new markets not currently being served. These opportunities may be in areas closely related to the existing business definition such as; diagnostics, post treatment services, enhanced in-hospital services etc. They could also include opportunities more diverse from the traditional private hospital business such as; aged care, preventative health care, 'wellness' and disease prevention services

The ability to make strategic change a reality will be dependant upon applied marketing knowledge and skills. As strategic priorities are established, detailed marketing objectives and strategies need to be formulated and implemented with cost accountability and key performance indicators used for tracking and monitoring progress.

Given the power distribution of key stakeholders in the industry, a key ongoing management knowledge and skill area will be the effective management of relationships with key supplier groups such as the medical practitioners, health insurance funds and equipment suppliers. Ongoing and proactive relationship planning and actions will be fundamental here so that issues are resolved in a timely and cost effective manor and to ensure that crisis or ad hoc solutions are avoided as much as possible.

Future research projects will look to quantify some of the dimensions uncovered in this exploratory research project to better understand how representative issues are that were mentioned by the respondents to this research.

\section{$\underline{\text { References }}$}

Australian Bureau of Statistics (ABS), 2004, ‘4390.0, Private Hospitals, Australia', Canberra

Australian Bureau of Statistics (ABS), 2006, ‘4390.0, Private Hospitals, Australia, 2004-05’, Release date July 14, 2006, Canberra 
Allee V( 2003), Evolving Business Forms for the Knowledge Economy. In Handbook on Knowledge Management (Holsapple CW, Ed), Vol 2, Knowledge Directions, 605622, Berlin

Ancori B, Bureth A and Cohendet P (2000), The Economics of Knowledge: The Debate About Codification and Tacit Knowledge, Industrial and Corporate Change, Vol 9, No 2, June

Baskerville R and Dulipovici A (2006), The Theoretical Foundations of Knowledge Management, Knowledge Management Research and Practice, 4, 83-105

Blumentritt R and Johnston R 1999, Towards a Strategy for Knowledge Management, Technology Analysis and Strategic Management, Vol 11, No 3, September 1, 287-300

Davenport T H and Prusk L (1998), Working Knowledge: How Organisations Manage What They Know, Harvard Business School Press, Boston.

De Holan P M, Phillips N and Lawrence T B (2004), Managing Organizational Forgetting, Sloan Management Review, Winter, Vol 45, No2, 45-51 Demarest M (1997), Understanding Knowledge Management, Long Range Planning, 30 (3), 374-384

Deutsche Bank, 2005, Private Hospital Operators: Consolidation Remains the Key to Earnings Growth, July 19

Drew S (1999), Building Knowledge Management into Strategy: Making Sense of a New Perspective, Long Range Planning, 32 (1), 130-136

Drucker P (1992), The New Society of Organisations, Harvard Business Review, September/October, 95-105

Gap Congress on Knowledge Capital (2005), Melbourne, Parliament House of Victoria, November 4

Foley M, 2000, “The Changing Private-Public Balance' in Health Reform in Australia and New Zealand, Ed Abby L Bloom, Oxford University Press, South Melbourne (99114)

Gedes and Grosset (1999), English Dictionary, David Dale House, New Lanark

Guptill J (2005), Knowledge Management in Health Care, Journal of Health Care Finance, Spring, Vol 31, Iss3, New York, 10-15

Hansen M T, Nohria N and Tierney T (1999), "What's Your Strategy for Managing Knowledge?”, Harvard Business Review, March-April (106-116)

Hassard J and Kelemen M (2002), Production and Consumption in Organisational Knowledge: The Case of the 'Paradigms Debate”, Organisation, 9 (2), 331-355 
Hedlund G (1999), The Intensity and Extensity of Knowledge and the Multinational Corporation as a Nearly Recomposable System, Management International Review, 39, 5

Inkpen A C 1996, Creating Knowledge Through Collaboration, California Management Review, Vol 39, Iss 1, 123-140

Jashapara A (2004), Knowledge Management: An Integrated Approach, Pearson Education, Harlow

Johnston R B and Gregor S 2000, A Theory of Industry- Level Activity for Understanding the Adoption of Interorganisational Systems, European Journal of Information Management, Vol 9, No 44, 243- 251

Low D A and Prior M, 2005, Private Hospital Operators, Deutsche Bank Industry Update, Asia Pacific Australia, July 19, 2005

Macquarie Research Equities (MRE), 2003, a), Ramsay Health Care Report, July

Mertins K, Heisig P and Vorbeck J (2000), "Knowledge Management: Best Practices in Europe”, Springer-Verlag, New York

Mowery D C, Oxley J E, Silverman B S, Grant R M and Spender J C, 1996, Strategic Alliances and Interfirm Knowledge Transfer, Strategic Management Journal, Vol 17, Winter, 77-91

Parise S and Henderson J C 2001, Knowledge Resource Exchange in Strategic Alliances, IBM Systems Journal: Knowledge Management, Vol 40, No 4

Perrott B E, 2005, Dynamics in the Australian Private Hospital Industry, UTS Working Paper Series 4/05, Sydney

Perrott B E (2006), “Knowledge Management in Health: An Evolution?”, Proceedings of the conference; Knowledge Management: The Key to Innovative Health Programs, March $8^{\text {th }}$ and $9^{\text {th }}$, Sydney

Pilzer, Paul Zane,2002, “The Wellness Revolution”, John Wiley and Sons Inc, New York

Polanyi M (1967), The Tacit Dimension, Doubleday, New York

Porter M E, 1980, Competitive Strategy: Techniques for Analysing Industries and Competitors, Free Press, New York

Quintas P, Lefrere P and Jones G (1997), Knowledge Management : A Strategic Agenda, Long Range Planning, 30 (3), 385-391

Ramsay Health Care Limited (2006), Annual Report, ABN 57001288768 
Reitzig M (2004), Strategic Management of Intellectual Property, Sloan Management Review, Spring, Vol 45, No 3 pp25-40

Rumelt R P, Schendel D and Teece D J, 1991, Strategic Management and Economics,Strategic Management Journal, Vol 12, pp 5-29

Swan J, Scarborough H and Preston J (1999), Knowledge Management- the next fad to forget people?", Proceedings of the $7^{\text {th }}$ European Conference on Information Systems, Copenhagen

Weigand W A and Davis D G (1994), Encyclopaedia of Library History, Garland, New York

Additional Interviews;

Interview with Patrick Grier, 2004, CEO, Ramsay Health Care, December, Sydney 